\title{
ELECTRICAL ENDOSMOSE. II
}

\author{
BY T. R. BRIGGS, H. S. BENNETT AND H. L. PIERSON
}

\section{The Apparatus}

After a careful study of the literature on electrical endosmose, the results of which have been communicated in a previous article, ${ }^{1}$ it was decided to construct an apparatus similar to the one used so successfully by Perrin. ${ }^{2}$ There was finally developed ${ }^{3}$ a modified electro-osmometer, possessing certain distinct advantages over Perrin's original apparatus. In the latter instrument there was no means of removing the gas that might be liberated from the electrodes on the passage of an appreciable current, and this gas, collecting in large bubbles beneath the vertical diaphragm, tended to cause a flow of liquid quite irrespective of electrical endosmose. In the apparatus to be described this difficulty was solved in a very simple way and there was added a somewhat novel means of measuring quantitatively the rate of flow through the diaphragm, by observing the speed with which an air bubble moved along a calibrated tube.

The electro-osmometer is constructed almost entirely of rather heavy glass and is essentially a horizontal tube with several vertical side arms. The complete apparatus is depicted in Figure I. The horizontal electrolyzing chamber containing the diaphragm is about three centimeters in diameter and thirty centimeters long; the two vertical electrode tubes $A_{1}$ and $A_{2}$ are about ten centimeters apart. In these tubes are placed the platinum electrodes $E_{1}$ and $E_{2}$ which are held in position by being sealed into narrow glass tubes supported in corks grooved so as to permit the gases generated during electrolysis to escape. Midway between $A_{1}$ and $A_{2}$, the horizontal electrolyzing chamber is enlarged slightly for

\footnotetext{
${ }^{1}$ Jour. Phys. Chem., 21, 198 (I917).

${ }^{2}$ Jour. Chim. phys., 2, 601 (1904).

${ }_{3}$ The early experiments were performed with the assistance of Mr. G. D. Kratz.
} 
a distance of four centimeters; this enlarged section forms the chamber in which the porous diaphragm is constructed. There is a small vertical side arm in the upper part of this enlarged section, in order that a thermometer may be inserted into the diaphragm.

At $R_{1}$ and $R_{2}$ the "bubble tube" is joined to the electroosmometer with short rubber connections. Attached to the horizontal chamber by ground glass joints, are two three-way cocks, $C_{1}$ and $C_{2}$, by the proper manipulation of which it is a simple matter to introduce the air bubble at $\mathrm{B}$. The speed

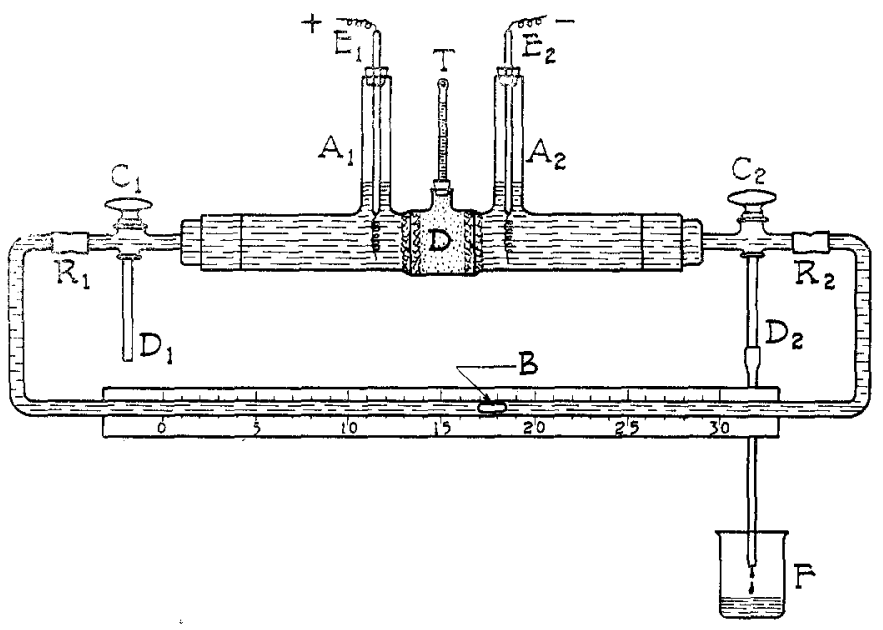

Fig. I

and direction of flow through the diaphragms are measured by the movement of this bubble, with the aid of a stopwatch and a scale divided into centimeters. Having determined the volume per centimeter of the bubble tube, one may calculate the volume of liquid transported in unit time through the diaphragm.

The air bubble is introduced into the measuring tube as follows: After the diaphragm has been built up in the chamber $\mathrm{D}$, the whole apparatus is assembled as shown in Figure $I$ and the measuring tube is connected to the osmometer at $R_{1}$ and $R_{2}$. The whole system is filled with the solu- 
tion to be employed by adding the liquid through the vertical arms $A_{1}$ and $A_{2}$ and properly manipulating the cocks at $C_{1}$ and $C_{2}$. Care is taken at this point to see that the exit tube $\mathrm{D}_{2}$ (including the rubber tube connected thereto) is completely filled with liquid.

The second exit tube $D_{1}$ is now placed beneath the surface of distilled water in a beaker, connection is made between $\mathrm{D}_{1}$ and the measuring tube through the three-way cock $\mathrm{C}_{1}$ and the exit tube $D_{2}$ is likewise connected to the measuring tube through $\mathrm{C}_{2}$. Owing to the siphon action of the long exit tube $\mathrm{D}_{2}$, distilled water is sucked through the measuring tube from $D_{1}$ and out at $D_{2}$, into the beaker at $F$. When the original (conducting) liquid has been displaced completely by distilled water, $C_{1}$ is closed and the surface in the beaker at $D_{1}$ is lowered until the end of $D_{1}$ is free. By carefully manipulating $\mathrm{C}_{1}$ an air bubble of the desired length is drawn into the exit tube $D_{1}$ and the latter is once again immersed in the beaker of water. On again opening $\mathrm{C}_{2}$, the bubble is carried along the measuring tube into any desired position. Finally, the level of the liquid in the vertical electrode chambers $A_{1}$ and $A_{2}$ is adjusted and the osmometer proper connected with the measuring tube through $C_{1}$ and $C_{2}$.

The most difficult operation is forming the diaphragm. The finely powdered and carefully sieved material is treated several times with the solution to be employed and is then introduced into the apparatus in the form of a paste. The actual diaphragm formation is carried out with the aid of suction as follows:

The body of the apparatus, minus the connecting parts, is placed with the electrolyzing chamber vertical and is connected to the suction pump through one side arm, $A_{1}$ or $A_{2}$, the other being closed with a rubber stopper. The diaphragm is built up on a perforated cork or rubber plate, upon which is laid a compact layer of glass wool or pure cotton. Glass wool is the best substance for the purpose because it forms a comparatively strong and rigid plug. The rubber plate is kept in place by means of a glass rod which is in turn held in 
position by a rubber stopper inserted into the lower end of the electrolyzing chamber. The procedure is simply that of filtering with suction on an improvised, perforated plate and the diaphragm is prepared quickly and washed easily, ${ }^{1}$ so long as the individual particles are not excessively small. On adding an upper plug of glass-wool or cotton, and after removing the perforated rubber plate, the diaphragm is complete.

By following this procedure it is easy to use the same diaphragm with a number of different solutions, for one is able to wash the diaphragm completely in a comparatively short time. This procedure as been followed throughout these experiments.

As I have observed already, the new electro-osmometer eliminates the possibility of gases evolved at the electrodes producing an apparent electro-osmotic flow. Special tests have proved beyond doubt that the free evolution of gas during the passage of the current has no measurable influence on the movement of the air bubble in the calibrated tube, so long as the evolution is not too copious and the electrode tubes are clean. Moreover, the air bubble being sensitive to extremely slight differences in level between the columns of liquid in $A_{1}$ and $A_{2}$ a very slight endosmotic flow through the diaphragm is rendered visible by a motion of the air bubble in one direction or the other.

In carrying out the experiments, one determines the time required for the bubble (having reached a maximum speed) to pass between two points on the scale, after which the direction of the currect is reversed and the time of transit in the opposite direction ascertained. After several readings, the mean is taken in case the temperature-as measured by the thermometer in the diaphragm-has not changed appreciably. From these observations the speed of the bubble is calculated in terms of centimeters per second. Likewise readings are taken of the temperature in the center of the

${ }^{1}$ Care must be taken never to suck air into the diaphragm. 
diaphragm and the voltage acrosss the electrodes. The subsequent calculations follow.

In the previous paper ${ }^{1}$ we saw that

$$
\mathrm{V}_{e}=\frac{q \mathrm{E} \epsilon \mathrm{D}}{4 \pi \eta l} \text {. }
$$

For a particular diaphragm of constant length and cross section, the volume of liquid $\mathrm{V}_{e}$ transported in unit time depends upon the potential drop through the diaphragm, the interface potential $\epsilon$, and the values of $D$ and $\eta$ for the liquid. Since $\epsilon, \mathrm{D}$ and $\eta$ are constant for a given solution at a particular temperature, under such circumstances the rate of flow through the diaphragm should be proportional to the voltage drop between the two extremes.

When an appreciable current of electricity flows through the diaphragm, however, Joule heating occurs and the temperature rises. At constant voltages, accordingly, $V_{e}$ will depend upon the variation of $\epsilon, \mathrm{D}$ and $\eta$ with the temperature. Since $\epsilon$ depends upon selective adsorption of ions and we know little regarding the way selective adsorption changes with the temperature, we cannot compute the temperature coefficient. It seems probable, however, that $\epsilon$ is approximately constant within narrow temperature limits if equilibrium is established and the ion composition of the liquid phase does not change with time. We know that the dielectric constant becomes smaller as the temperature increases. At $0^{\circ}, \mathrm{D}$ for water is 88 ; at $\mathrm{r} 7^{\circ}$ it is $8 \mathrm{I}$. If we call $\mathrm{I} / \eta$ the fluidity of the liquid, the increase in the fluidity with the temperature is known for pure water or very dilute solutions and $V_{e}$ is directly proportional to the fluidity.

Theoretically, the temperature coefficient of the fluidity is the predominating factor and one would expect $V_{e}$ to increase strongly with the temperature. This is actually the case. But one should not expect the flow to keep pace with the increased fluidity of the liquid since the temperature coefficient of $D$ is negative. Accordingly, it is not strictly accurate to assume that the rate of endosmose is inversely pro-

1 Jour. Phys. Chem., 2I, 201 (I9I7). 
portional to the viscosity, though for ordinary purposes this way of putting it is sufficiently close to the truth. These conclusions have been subjected to experimental test, in the manner to be described.

\section{Varying the Voltage at Constant Temperature}

Some glass tubing was pulverized and sieved, the portion passed by an 80 -mesh sieve but retained by a $\mathbf{I} 20$ one being used to make a diaphragm. The diaphragm was built up in the usual manner and retained in position by plugs of glass wool. Distilled water was used as the liquid. To obtain a wide range of voltages, the current from a battery of storage cells ( 32 volts) was passed through a theostat frame and the field coils of a small o. I $\mathrm{kw}$ generator, operated by an induction motor. By changing the current in the field coils the potential of the transformed current could be increased to 500 volts as a maximum.

After several preliminary experiments had been made by Mr. H. S. Bennett, to standardize conditions, the data of Table I were obtained.

\section{TABLE I}

Influence of Applied Potential on Rate of Flow Glass diaphragm in distilled water. Liquid flowed to cathode

\begin{tabular}{|c|c|c|c|c|c|}
\hline $\begin{array}{l}\text { Applied } \\
\text { potential } \\
\text { (Volts) }\end{array}$ & $\begin{array}{l}\text { Time } \\
\text { (Secs.) }\end{array}$ & $\begin{array}{c}\text { Distance } \\
\mathrm{Cm}\end{array}$ & Temp. & $\begin{array}{l}\text { Rate. Cm } \\
\text { per second }\end{array}$ & Rate/potential \\
\hline 530 & I $3 \cdot 5$ & 20 & I9.8 & I. 48 & $27.9 \times \mathrm{IO}^{-4}$ \\
\hline 500 & I3.8 & 20 & 19.9 & I .45 & $29.0 \times 10^{-4}$ \\
\hline $45^{\circ}$ & 5.2 & 20 & I9.8 & I .32 & $29.3 \times 10^{-4}$ \\
\hline 416 & I6. I & 20 & 20.0 & I. 24 & $29.8 \times 10^{-4}$ \\
\hline $35^{\circ}$ & I9. I & 20 & 20.5 & I. .05 & $30.0 \times 10^{-4}$ \\
\hline 300 & 22.2 & 20 & 19.7 & 0.90 & $30.0 \times 10^{-4}$ \\
\hline 250 & I 3.2 & IO & I9.6 & 0.76 & $30.4 \times 10^{-4}$ \\
\hline 200 & I 6.8 & 10 & 19.7 & 0.60 & $30.0 \times 10^{-4}$ \\
\hline I 50 & 22.0 & IO & 19.6 & 0.45 & $30.0 \times 10^{-4}$ \\
\hline 100 & 32.8 & IO & 19.5 & 0.30 & $30.0 \times 10^{-4}$ \\
\hline
\end{tabular}


The data are shown graphically in Figure 2 by means of a curve drawn between bubble speed and applied potentials. The curve is a straight line except at very high voltages where the speed of the bubble was fairly great and the resistance

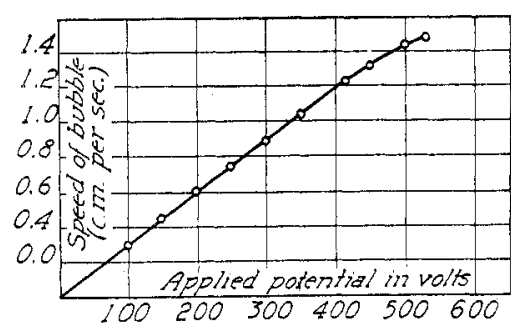

Fig. 2 to the flow of liquid through the apparatus began to complicate matters. A number of minor tests were also made. The length of the air bubble acting as indicator in the measuring tube was increased very considerably without appreciably affecting the speed. The diaphragm itself was next replaced by a solid plug, to ascertain whether the motion of the bubble might be due to something independent of electrical endosmose through the porous diaphragm; on applying r Io volts no motion at all occurred.

\section{Varying the Temperature at Constant Voltage}

Measuring the temperature coefficient of electrical endosmose proved to be less easy. Some data obtained for me by Mr. H. L. Pierson, in Worcester (Worcester Polytechnic Institute), were fairly satisfactory. Alundum and carborundum diaphragms were used and $\mathrm{V}$ increased steadily with the temperature. When we came later to renew the work in the Cornell laboratory, we tried powdered glass and got very irregular results. Mr. Bennett found at first a maximum value for the bubble speed as the temperature rose from $20^{\circ}$ to $65^{\circ}$, seeming accordingly, to corroborate the testimony of Cruse. ${ }^{1}$

On allowing the apparatus and its contents to cool, it was found on repeating the test that the rate of flow was now very different from what it had been at the beginning of the

${ }^{1}$ Phys. Zeit., 6, 201 (1905). 
experiment. There was a marked decrease even though the same voltage was applied. The current flowing between the electrodes was measured on a milliammeter and showed a pronounced increase, which grew rapidly when the temperature was kept for a time at $60^{\circ}$. The glass of the plugs and diaphragm appeared to dissolve and the ion composition of the liquid-which was originally distilled water or dilute alkali-changed appreciably. The adsorption equilibrium between liquid and diaphragm could no longer be regarded as constant and the rate of endosmose fell off. The maximum obtained in the first run was explained; at temperatures below the maximum, the increasing fluidity had more influence than the increasing ion concentration, while above the maximum point the reverse was true. This explanation is based upon the fact that the solid dissolved faster at high temperatures than at low temperatures.

After keeping the diaphragm in contact with the hot solution for several hours, then cooling and allowing the whole to stand overnight, the heating test was repeated. No maximum whatever was observed between the same temperatures; the rate of endosmose increased steadily with rising temperature, but did so much less rapidly than the fluidity.

Crystolon (carborundum) and alundum diaphragms held in position with glass wool gave irregular results also, so glass was eliminated as far as possible, and washed asbestos or macerated filter paper substituted. Distilled water was used as liquid, and was kept in contact with the diaphragm for 24 hours to insure equilibrium. The diaphragm was heated from the outside by pouring a steady stream of water from a heater over the central portion of the endosmometer. A special test showed that the speed of the bubble was not affected by having hot or cold water in the measuring tube. The data follow, the values of time recorded in the table being the mean of at least six readings in every case. 
264 T. R. Briggs, H. S. Bennett and H. L. Pierson

TABLE 2

INFLUENCE OF TEMPERATURE AT CONSTANT VOLTAGE Asbestos diaphragm. Distilled water. Flow to cathode

\begin{tabular}{|c|c|c|c|c|c|}
\hline $\begin{array}{l}\text { Applied } \\
\text { potential } \\
\text { (Volts) }\end{array}$ & $\begin{array}{l}\text { Time } \\
\text { (Secs.) }\end{array}$ & $\begin{array}{c}\text { Distance } \\
(\mathrm{Cm})\end{array}$ & $\begin{array}{c}\text { Tempera- } \\
\text { ture } \\
\left({ }^{\circ} \mathrm{C}\right)\end{array}$ & $\begin{array}{l}\text { Rate. Cm } \\
\text { per second }\end{array}$ & $\begin{array}{l}\text { Rate times } \\
\text { viscosity }\end{array}$ \\
\hline 250 & I 6.2 & 5 & $18.0^{\circ}$ & 0.308 & $32 \times 10^{-4}$ \\
\hline 250 & $25 \cdot 9$ & IO & $30.5^{\circ}$ & 0.386 & $3 \mathrm{I} \times \mathrm{IO}^{-4}$ \\
\hline 250 & 22.0 & IO & $39.0^{\circ}$ & 0.454 & $30 \times \mathrm{IO}^{-4}$ \\
\hline 250 & I9. 8 & IO & $46.5^{\circ}$ & 0.505 & $30 \times 10^{-4}$ \\
\hline 250 & 18.0 & IO & $58.0^{\circ}$ & 0.588 & $30 \times 10^{-4}$ \\
\hline \multicolumn{6}{|c|}{ Cellulose in boiled water (air free) } \\
\hline 400 & 28.2 & 5 & $22.6^{\circ}$ & 0.177 & I6.7 $6 \mathrm{IO}^{-4}$ \\
\hline 400 & $25 \cdot 5$ & 5 & $27.6^{\circ}$ & 0.196 & $16.7 \times 10^{-4}$ \\
\hline 400 & $22 \cdot 3$ & 5 & $36.1^{\circ}$ & 0.224 & I $5.9 \times$ Io $^{-4}$ \\
\hline 400 & I9. I & 5 & $52.0^{\circ}$ & 0.262 & $\mathrm{I} 4.0 \times 10^{-4}$ \\
\hline 400 & $\mathrm{I} 7 \cdot \mathrm{I}$ & 5 & $67.0^{\circ}$ & 0.292 & $\mathrm{r} 2.5 \times \mathrm{IO}^{-4}$ \\
\hline
\end{tabular}

The following data with a crystolon (CSi) diaphragm had previously been obtained by Mr. Pierson at Worcester with the same apparatus:

TABLE 3

200-Mesh Crystolon in $\mathrm{M} / 5 \mathrm{OO} \mathrm{NaOH}$. Flow to cathode

\begin{tabular}{c|c|c|c|c|c}
\hline $\begin{array}{c}\text { Applied } \\
\text { potential } \\
\text { (Volts) }\end{array}$ & $\begin{array}{c}\text { Time } \\
\text { (Secs.) }\end{array}$ & $\begin{array}{c}\text { Distance } \\
\text { (Cm) }\end{array}$ & $\begin{array}{c}\text { Tempera- } \\
\text { ture } \\
\left({ }^{\circ} \mathrm{C}\right)\end{array}$ & $\begin{array}{c}\text { Rate. Cm } \\
\text { per sec. }\end{array}$ & $\begin{array}{c}\text { Rate times } \\
\text { viscosity }\end{array}$ \\
\cline { 1 - 5 } I IO & 53 & 5 & $23.5^{\circ}$ & $0.087^{\circ}$ & $8.1 \times$ IO $^{-4}$ \\
I IO & 49 & 5 & $27.0^{\circ}$ & 0.093 & $8.0 \times$ IO $^{-4}$ \\
I IO & 47 & 5 & $29.0^{\circ}$ & 0.097 & $8.0 \times$ IO $^{-4}$ \\
I IO & 44 & 5 & $32.5^{\circ}$ & 0.103 & $7.9 \times 10^{-4}$ \\
I IO & 45 & 5 & $33.5^{\circ}$ & 0.101 & $7.6 \times 10^{-4}$ \\
IOO & 43 & 5 & $35.5^{\circ}$ & 0.106 & $7.6 \times 10^{-4}$ \\
IOO & 46 & 5 & $36.5^{\circ}$ & 0.109 & $7.7 \times 10^{-4}$ \\
IOO & 40 & 5 & $38.5^{\circ}$ & 0.114 & $7.8 \times 10^{-4}$ \\
IO3 & 44 & 5 & $38.5^{\circ}$ & 0.110 & $7.5 \times 10^{-4}$ \\
I IO & 38 & 5 & $40.5^{\circ}$ & 0.120 & $7.8 \times 10^{-4}$ \\
I IO & 37 & 5 & $41.5^{\circ}$ & 0.122 & $7.8 \times 10^{-4}$ \\
IO4 & 38 & 5 & $45.5^{\circ}$ & 0.126 & $7.4 \times 10^{-4}$
\end{tabular}

If the rate of endosmose varied with the fluidity of the liquid and with nothing else, the product of rate into the co- 
efficient of viscosity should be constant. Values of this product are given in the last column of data. These numbers decrease slightly as the temperature rises and the rate of decrease is more rapid apparently, the higher the temperature. The data show that some other factor comes into play and tends to counteract slightly the effect of rising fluidity in increasing the rate of flow. We have seen that this factor is theoretically the dielectric constant, to which the rate is proportional; since this decreases with rising temperature, we can account for the data recorded in the last column of Tables 2 and 3 .

Nevertheless at room temperature and for moderate temperature changes the rate of flow is approximately proportional to the fluidity of the liquid. Assuming that the very dilute solutions employed in this work have the same viscosity as pure water, one can correct for temperature changes at constant voltage as follows:

$$
\operatorname{RATE}_{20^{\circ}}=\frac{\eta_{t^{\circ}}}{\eta_{20^{\circ}}} \text { times } \operatorname{RATE}_{t^{\circ}} \text {. }
$$

Since $\eta_{20^{\circ}}=0.0100$ dyne per $\mathrm{cm}^{2}$ almost exactly, the rates corresponding to $20^{\circ}$ are very easily calculated from the rate determined at experimental temperatures.

The voltage correction is made in a similar manner. The rate corresponding to roo volts may be computed as follows since at constant temperature

$$
\operatorname{RATE}_{(100 \text { volts })}=\frac{\text { IOO }}{\mathrm{E}} \text { times } \mathrm{RATE}_{(\mathrm{E} \text { volts })}
$$

This reduction has been carried out in Table 3 .

By combining (3) and (4) we obtain the composite expression which allows the rate corresponding to $20^{\circ}$ and 100 volts to be computed.

$$
\text { CORRECTED RATE }=\frac{\text { IOO } \eta_{t} \circ \text { times OBSERVED RATE }}{E \eta_{20^{\circ}}}
$$

Or, when water or dilute aqueous solutions are used, remembering that $\eta_{20^{\circ}}=0$.oroo dyne per $\mathrm{cm}^{2}$, nearly

$$
\text { CORRECTED RATE }=\text { OBSERVED RATE times } \frac{\eta_{t^{\circ}} \times 10^{4}}{\mathrm{E}} \text {. }
$$




\section{Acid and Alkaline Solutions}

A set of experiments was made under my direction by Mr. Pierson in Worcester, for the sake of duplicating approximately Perrin's work with. dilute solutions of acids and bases. The degree of refinement of the work was by no means the maximum possible with the apparatus but the results obtained were quite satisfactory. The Norton Company was kind enough to furnish us with graded and purified samples of crystolon and alundum, with which we worked. The data which follow were obtained with a diaphragm of crystolon (120-mesh). The concentration of the solutions was determined only approximately and no great care was taken to use sodium hydroxide absolutely free from carbonates.

TABLE 4

Acid and Alkaline Solutions

\begin{tabular}{|c|c|c|c|}
\hline Solution & $\begin{array}{l}\text { Concentra- } \\
\text { tion }\end{array}$ & $\begin{array}{c}\text { Average rate (corrected) } \\
\text { Cm per sec. }\end{array}$ & $\begin{array}{l}\text { Direction of } \\
\quad \text { flow }\end{array}$ \\
\hline Water (distilled) & 一 & O. II 4 & to cathode \\
\hline $\mathrm{NaOH}$ & $N / 500$ & 0.217 & to cathode \\
\hline $\mathrm{NaOH}$ & $N / 250$ & 0.198 & to cathode \\
\hline $\mathrm{NaOH}$ & $N /$ IOO & 0.154 & to cathode \\
\hline Water (distilled) & - & 0.159 & to cathode \\
\hline $\mathrm{HCl}$ & $N /$ IOOO & 0.070 & to anode \\
\hline $\mathrm{HCl}$ & $N / 500$ & 0.102 & to anode \\
\hline $\mathrm{HCl}$ (check) & $N / 500$ & 0.099 & to anode \\
\hline $\mathrm{HCl}$ & $N /$ IOO & 0.074 & to anode \\
\hline
\end{tabular}

Figure 3 illustrates these data. In the experiments the same diaphragm was used throughout, for by arranging the apparatus as described previously, the diaphragm was washed easily and completely with water and solution at $50^{\circ}$ or $60^{\circ}$. The first washing required more than a liter of hot water and this was followed with the solution to be studied, hot at first and then cold. The rates tabulated above represent the averages of many readings and have been reduced to Ioo volts and $20^{\circ}$ by means of Equation 5 .

The results confirm Perrin completely and the curve is 
similar to the one drawn by Freundlich ${ }^{1}$ from Perrin's data. The reversal is very sharply defined and the isoelectric point lies over on the acid side at concentrations of $\mathrm{HCl}$, which are less than one thousandth normal. No allowance has been made for carbonates in the sodium hydroxide, although very pure alkali was used originally, and the water was ordinary distilled water of excellent quality.

On studying alundum a sharp acid-alkali reversal was found. Although these diaphragms were held in place with small plugs of glass wool, the trouble caused thereby was much

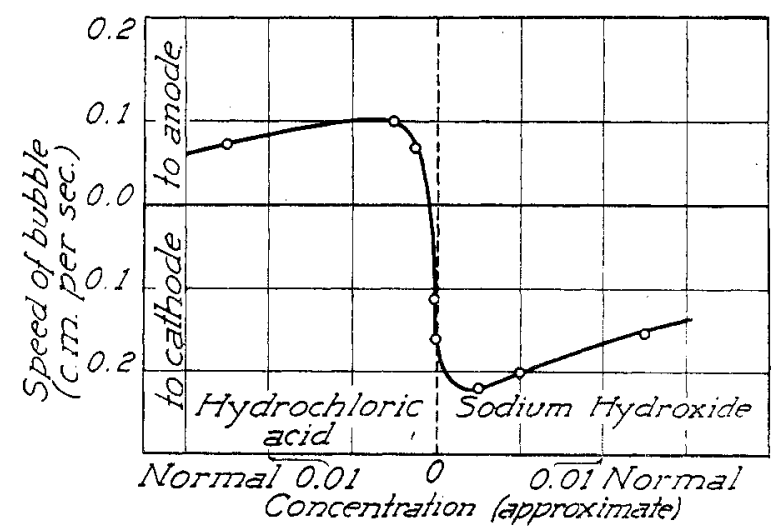

Fig. 3

smaller than when the temperature-effects were studied, doubtless because the temperature in the diaphragm was kept low and possibly because the glass was of better quality. At any rate, no current was observed in the milliammeter and a diaphragm of glass wool alone showed practically no endosmose. When powdered glass was used, however, there was always a strong flow to the cathode and no reversals were obtained in acid solutions-not even in $N /$ Ioo hydrochloric acid. As the acid content increased, the flow to the cathode approached zero. On the other hand, reversals were obtained with diaphragms of gelatine and agar jellies.

The Volume of Solution Transported

Having made these measurements, Mr. Pierson ascertained the average volume per linear centimeter of the measur-

1 "Kapillarchemie," 236 (r9o9). 
ing tube containing the bubble. Experiment gave $0.125 \mathrm{cc}$ per centimeter. For $N / 5 \mathrm{OO} \mathrm{NaOH}$ in Table 4 , the bubble rate was $0.217 \mathrm{~cm}$ per second or $13 \mathrm{~cm}$ per minute, corresponding to $\mathrm{I} .6 \mathrm{cc}$ for a potential drop of about $\mathrm{I} 2$ volts per centimeter through the diaphragm. With his apparatus Perrin rarely obtained more than $\mathrm{O}$. I cc per minute with a potential gradient of ro volts per centimeter.

\section{Polyvalent Ions}

A long series of experiments was carried out to duplicate, if possible, the data obtained by Perrin; the results confirmed in all the essentials what he found. For the particular salts studied, the so-called valence rule held satisfactorily. Barium was used as a typical divalent cation, sulphate, ferrocyanide and ferricyanide as anions. In alkaline solution adsorbed barium ions reduced the rate of endosmose and did so very much more strongly than did equivalent quantities of the alkali metals. In acid solution, barium was far less active, it being the polyvalent anions which reduced the endosmose most strongly.

\section{Copper Salts}

Using an alundum diaphragm, Mr. Pierson obtained some interesting results with solutions of certain copper salts. His data follow:

TABLE 5

COPPER SALTS

\begin{tabular}{|c|c|c|c|}
\hline Solution & Normality & $\begin{array}{c}\text { Rate (corrected) } \\
\mathrm{Cm} / \text { second }\end{array}$ & Direction \\
\hline Copper acetate & O.I & 0.048 & to anode \\
\hline Copper acetate & $0 . \mathrm{OI}$ & 0.085 & to anode \\
\hline Copper acetate & 0.004 & 0.098 & to anode \\
\hline Copper acetate & 0.002 & $0.09 \mathrm{I}$ & to anode \\
\hline Copper nitrate & 0.1 & $0.08 \mathrm{I}$ & to anode \\
\hline Copper nitrate & 0.01 & 0.127 & to anode \\
\hline Copper nitrate & 0.004 & 0.132 & to anode \\
\hline Copper nitrate & 0.002 & O. II I & to anode \\
\hline Copper sulphate & 0.2 & 0.019 & to cathode \\
\hline Copper sulphate & $0 . \mathbf{I}$ & 0.020 & to cathode \\
\hline Copper sulphate & O.OI & 0.016 & to anode \\
\hline Copper sulphate & 0.004 & 0.028 & to anode \\
\hline Copper sulphate & 0.002 & 0.089 & to anode \\
\hline Copper chloride & 0.002 & 0.113 & to anode \\
\hline
\end{tabular}


The curves of Figure 4, illustrating these data, show clearly how the preferential adsorption of ions may change with the concentration. In many respects the data agree with Coehn's ${ }^{1}$ except that he states that copper sulphate solutions always go to the cathode. We found a reversal as the concentration decreased, very dilute solutions flowing

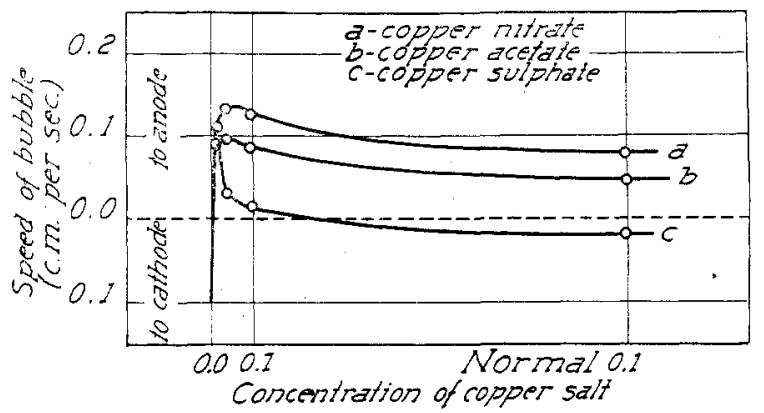

Fig. 4

to the anode. Of course hydrolysis must be a disturbing factor. Leaving this out of account, copper as ion seems to be absorbed in preference to acetate, nitrate, chloride and sulphate in dilute solutions, but less readily than sulphate as the solution becomes more concentrated. These data account for the fact that copper sulphate solutions go to the cathode through a porous cup and copper nitrate solutions go to the anode.

\section{Electrical Endosmose and Dyeing}

Bancroft ${ }^{2}$ has discussed the theory of dyeing with acid and basic dyes from the view-point of selective adsorption. So far as known, the adsorption of a dye by fibers or mordants depends, for a given adsorbent, upon the nature of the dye, its concentration, the other substances present in the dye bath, as well as upon the temperature. A basic dye is one having a colored basic radical, and an acid dye is one having

1 Zeit. Elektrochemie, I6, 586 (I910).

${ }^{2}$ Jour. Phys.' Chem., I8, I, I I8, etc. (I9I4). 
a colored acid radical. For the particular case of an acid dye, we have the following rules:

"( $\mathrm{I})$ The dye is taken up most readily in an acid solution but may be taken up in a neutral or alkaline solution.

(2) A readily adsorbed anion decreases the amount of dye taken up.

(3) A readily adsorbed cation increases the amount of dye taken up."

For the case of a basic dye, the same rules hold, if one substitutes "alkaline" for "acid" and "cation" for "anion," and vice versa. A basic dye is taken up most readily in an alkaline solution. After all, rule ( $\mathbf{r}$ ) above is only a special case covered by (2) and (3) and would fail to hold in case the hydrogen ion of a particular acid were less readily adsorbed than its anion.

Mr. Pierson carried out a series of experiments designed to test the first of these generalizations. Alundum diaphragms were used and the following data obtained:

TABLE 6

Acid and Basic Dyes

\begin{tabular}{|c|c|c|c|c|}
\hline Dye & $\begin{array}{l}\text { Nature } \\
\text { of dye }\end{array}$ & $\begin{array}{l}\text { Solution } \\
\text { (approx.) }\end{array}$ & $\begin{array}{l}\text { Rate (correc- } \\
\text { ted). } \mathrm{Cm} / \mathrm{sec} .\end{array}$ & Direction \\
\hline Eosine & acid & $N / 500 \mathrm{HCl}$ & (very slow) & ? \\
\hline Eosine & acid & $N / 500 \mathrm{NaOH}$ & 0.092 & to cathode \\
\hline Crystal ponceau & acid & $N / 500 \mathrm{HCl}$ & (very slow) & : \\
\hline Crystal ponceati & acid & $N / 500 \mathrm{NaOH}$ & 0.059 & to cathode \\
\hline Safranine & basic & $N / 500 \mathrm{HCl}$ & 0.077 & to anode \\
\hline Safranine & basic & $N / 500 \mathrm{NaOH}$ & (very slow) & $?$ \\
\hline Methylene blue & basic & $N / 500 \mathrm{HCl}$ & 0.022 & to anode \\
\hline Methylene blue & basic & $N / 500 \mathrm{NaOH}$ & (no flow) & \\
\hline
\end{tabular}

(In each case concentration of dye was approximately $\mathrm{N} / 5000$.)

The results are quite in accord with the theory, and they emphasize certain points, particularly the marked effects of which small amounts of dye may produce and the difference between soluble acid and basic dyes. Alundum behaves as mordant essentially like hydrous alumina, except that the amount of dye removed by alundum is small and the dyed 
material is not fast to washing. When crystal ponceau was used in alkaline solution it was easily removed from the alundum by washing; after the dye was used in acid solution, however, it could be washed out only with difficulty. Methylene blue displayed the opposite behavior, for it was washed out easily after it had been used in acid solution but was removed with difficulty after it had been used with alkali.

The experiment is an excellent illustration of the neutralization of adsorbed ions. Alundum adsorbs hydrogen ions from an acid solution and becomes charged positively. If one adds a basic dye, neutralization of the adsorbed hydrogen can occur only by the adsorption of the colorless acid radical (usually chloride), which we have found by experiment to be only slightly absorbed. Some neutralization does occur, because the concentration of anions has been increased by adding the dye. But when we add an acid dye, the colored acid radical is adsorbed strongly and the positive charge on the diaphragm is neutralized. This neutralization is indicated by the fact that the solution no longer flows to the anode in acid solutions as it should do normally, but becomes almost stationary. Likewise, the adsorption of dye base from an alkaline solution is another example of the neutralization of adsorbed ions (in this case adsorbed hydroxyl).

The results of this paper may be summarized as follows:

(I) A new and convenient apparatus has been devised for studying electrical endosmose.

(2) The rate of electrical endosmose is proportional directly to the applied potential, other conditions being kept unchanged.

(3) The rate of electrical endosmose increases with rising temperature. It is not exactly proportional to the fluidity of the liquid but increases slightly less rapidly than the fluidity does with rising temperature. The results of experiment confirm the equation

$$
\mathrm{V}_{e}=\text { constant times } \frac{\mathrm{ED}}{\eta}
$$

for a given diaphragm and a given liquid. 
272 T. R. Briggs, H. S. Bennett and H. L. Pierson

(4) Perrin's data have been confirmed.

(5) Electrical endosmose has been studied with solutions of certain copper salts. Copper acetate, nitrate, and chloride flow to the anode, showing that copper as ion is adsorbed more than acetate, nitrate or chloride, but reversal occurs to the cathode with copper sulphate, indicating that sulphate is adsorbed more strongly than copper as ion at all ordinary concentrations.

(6) The relation of dyeing to contact electrification has been made evident by experiments on electrical endosmose.

Cornell University 